\title{
User Workshop on High-Power Lasers at the Linac Coherent Light Source
}

\author{
Roger Falcone, Siegfried Glenzer, Dr. Stefan Hau-Riege
}

A two-day international workshop on the physics and integration of high-power lasers with the Linac Coherent Light Source was held in Stanford, USA, on October 1-2, 2013. The workshop was co-organized by UC Berkeley, Lawrence Berkeley, Lawrence Livermore and SLAC National Accelerator Laboratories. More than 150 scientists, including 30 students and postdocs who are working in high-intensity laser-matter interactions, fusion research, and dynamic high-pressure science came together from North America, Europe and Asia. The group discussed the most promising and important new physics experiments that will be enabled by the unique combination of high-power lasers with the world-class LCLS freeelectron laser X-ray beam.

High-power laser facilities across the world can now reach petawatt $\left(10^{15} \mathrm{~W}\right)$ laser power to drive laser targets into an extreme matter state. In this environment, the material at the laser focus is heated to high temperatures, which drive high-pressure high-density shocks, nuclear processes, as well as large magnetic and electric fields. These experiments hold great promise for laser-based acceleration of protons, electrons and ions to high energies, and for creating anti-matter and fusion neutrons. Furthermore, these processes produce secondary ultrafast $\mathrm{X}$-ray and $\gamma$-ray emissions.

The LCLS X-ray beam will enable novel research projects that take advantage of its unique in situ probing capability to help understand the plasma and material physics within the primary laser target, and to optimize the production of laser-accelerated particles and radiation. In addition, the laserproduced radiation is itself of great interest for heating matter for a wide range of applications, including fusion and cancer research. LCLS will be able to measure these ultrafast interaction processes in secondary targets. A third class of experiments will combine LCLS with laser driven X-rays for performing X-ray pump/X-ray probe measurements with complementary spectra, thus helping to resolve the ultrafast evolution of the physical properties of matter.

In four oral presentation sessions, one poster and one discussion session, workshop attendees developed the most promising high-impact science projects for the Matter in Extreme Conditions (MEC) instrument at LCLS. There was particular emphasis on prioritizing new laser, diagnostic and target developments for MEC. In addition, new collaborations were established to best use MEC's existing and future high-power laser capabilities.

At present, the MEC instrument combines the LCLS X-ray beam with two nanosecond Joule-class lasers that can irradiate targets at intensities approaching $10^{14} \mathrm{~W} / \mathrm{cm}^{2}$, which can launch strong shocks or ramp-compress solids to high pressures. Of particular interest for the workshop was MEC's short-pulse laser, which will be upgraded twice during 2014: first to $25 \mathrm{TW}(1 \mathrm{~J}, 40 \mathrm{fs}, 5 \mathrm{~Hz}$ ) and then to $200 \mathrm{TW}(8 \mathrm{~J}, 40 \mathrm{fs}, 0.002 \mathrm{~Hz}$ ) peak power.

The workshop's first oral presentation session was devoted to recent experimental results obtained at MEC during its first 12 months of operation. The speakers (H. J. Lee, A. Gleason, L. B. Fletcher, B. Nagler, G. Monaco, A. Ravasio and S. Vinko) presented highly successful new experimental studies. Generally, the experiments can be classified into four classes that have used LCLS x-ray probes to characterize extreme-matter states that were produced with the nanosecond beams or by LCLS itself. These include: 1) ultrafast diffraction measurements of phase transitions in materials of interest to fusion, earth and planetary sciences; 2) spectrally resolved x-ray Thomson scattering of Compton, plasmon and ion acoustic features; 3) absorption and fluorescence spectroscopy, and 4) phase-contrast imaging of shocks and ultrafast phenomena.

During these talks, the uniformity of the laser spots was brought up, because it can affect the interpretation of experiments that require large samples. Equivalent-plane cameras were suggested for future experiments to enable users to measure the laser-beam uniformity at the plane of the target and to determine the evolving laser beam's phase changes from shot to shot. In addition, attendees recommended as desirable improvements increasing the total laser energy on target, laser pulse shaping and shot-by-shot pulse monitoring.

Raising these issues has had immediate impact. Shortly after the workshop, the LCLS laser engineering team demonstrated ramplaser pulse shapes that were used successfully in material compression experiments. In addition, the team recently increased laser energy on target from $4.5 \mathrm{~J}$ to $6 \mathrm{~J}$ in $3 \mathrm{~ns}$ long pulses.

The first workshop day concluded with an update on technologies for achieving highpower laser pulses for future high-field research and a poster session. The latter provided an opportunity for students and young researchers to present their work and ideas for novel experiments at LCLS.

The poster session further highlighted the importance of first using theory and simulations to explore the most interesting science questions computationally and then to field controlled experiments. The highpower laser-matter interaction regime is theoretically and computationally very challenging. Particle speeds approach the speed of light, so fluid-hybrid schemes often do not apply. Kinetic modeling, like a particle-in-cell (PIC) scheme, is often required.

An example of a successful simulation approach employs adaptive particle refinement strategies that allow researchers to perform full-scale 2D simulations of an experiment at the required resolution with about 1 million CPU-hours. This can be accomplished in an adaptive scheme by creating additional simulation particles and randomizing them during the simulation.

\section{Published in Synchrotron Radiation News.}


Opening the second day of the workshops were SLAC director C. C. Kao, DOE Fusion Energy Sciences program manager A. Satsangi, and LCLS interim director U. Bergmann. After his warm welcome, Kao described the competitive landscape and access opportunities that use LCLS for research. Satsangi pointed out potential future funding opportunities for academic work at MEC through Fusion Energy Science programs, High Energy Density Laboratory Plasma Joint Program and the NSF/DOE Partnership. Bergmann reviewed recent science opportunities at all six LCLS instruments. He pointed out that MEC received its highest number of new proposals for Run 9: 35 out of 195 total for LCLS. He expects a success rate of $20-25$ percent. $\mathrm{He}$ also challenged the MEC community to evaluate a single-pulse kicker option for increased access to the LCLS beam through a shared-operation mode.

The second oral presentation session consisted of two parts, each discussing frontier research in high-intensity lasermatter interactions. The speakers of the first part (T. Ditmire, K. Krushelnik, B. M. Hegelich) pointed towards a bright future with opportunities for transformative discoveries by using LCLS as a new precision tool for controlling and optimizing the production of radiation sources with lasers incident on thin films at intensities of $10^{18}-10^{21} \mathrm{~W} / \mathrm{cm}^{2}$. This class of experiments will allow producing $>100 \mathrm{MeV}$ protons for possible cancer therapies or for isochoric heating studies of matter, such as testing the equation of state of dense matter. Other radiation sources can drive intense $\mathrm{x}$-ray sources or energetic electrons and positrons. In the talks, the speakers showed new experimental results from the Texas PetaWatt and the Michigan Hercules lasers. These high-power lasers' temporal and spatial beam quality has been key for them becoming efficient and stable sources. It was also suggested that the MEC's first laser upgrade include a plasma mirror to obtain a low prepulse level, which would be important for generating $\mathrm{MeV}$ proton beams.

The speakers of the second part (E. I. Moses, D. H. Froula, T. Tajima and M. Roth) focused on laser-produced radiation and particles. While Moses presented encouraging new progress towards achieving fusion conditions by inertial confinement fusion on the National Ignition Facility, the direct irradiation of matter by high-power laser-produced radiation sources has recently received wide attention for thermodynamic studies of heating, heat transport and ion stopping.

The third session described ongoing and future high-power laser developments that are particularly relevant for LCLS. In 2016, the Helmholtz International Beamline for Extreme Fields (HIBEF) instrument expects to commission a $200 \mathrm{TW}, 10 \mathrm{~Hz}$ laser combined with an $\mathrm{x}$-ray free electron laser beam for user experiments at XFEL at DESY in Germany. In addition, a kilojoule class laser is being planned for studying strong shocks. Another important thrust area is the European Light Infrastructure (ELI) project, where multiple sites support a range of attosecond, high-energy-density and laserdriven particle beams. Here, multiple PW laser beams at one site will allow pumpprobe studies with laser-generated x-rays.

These projects particularly benefit from lasers that can deliver high peak power, high energy and high average power. Lawrence Livermore National Laboratory is currently developing lasers projected to deliver $30 \mathrm{~J}, 30$ fs, $10 \mathrm{~Hz}, 130 \mathrm{~kW}$. Other high-averagepower laser projects for $\mathrm{x}$-ray free electron lasers are being pursued at the Rutherford Appleton Laboratory in the UK, and at the Helmholtz Zentrum Dresden-Rossendorf in Germany.

At the workshop's final oral presentation session (P. Chen, J. Wark) discussed new directions in high-energy-density physics enabled by combining high-power lasers and free electron laser sources. The speakers showed a detailed study of phase transitions in solids at high pressure and explored new experiments to measure quantum electron dynamics effects, where lasers allow studies with a strong accelerating force..

The workshop concluded with a discussion session. The speakers and discussion leaders (W. Goldstein, P. Heimann, A. Thomas, M. Wei, R. P. Drake,
S. Hau-Riege, P. Norris, W. White) touched many areas, such as prospects for students and young researchers, access to the MEC lasers, and hardware needs to perform cutting edge research at MEC.

Goldstein presented recent work performed by successful young researchers in the field of high-energy-density science and explained the importance to continue providing opportunities in this area Heimann followed this presentation briefly reviewing the MEC schedule and capabilities and describing access to MEC lasers. He announced a call for laser-only experiments at MEC aimed at demonstrating capabilities that may subsequently be applied in experiments combined with the LCLS X-ray beam. Shortly after the workshop, this call was announced on the LCLS website, and all workshop participants were notified.

The speakers then summarized input that they had received during the time leading up to the workshop, particularly concerning the areas of diagnostics, targets, and optics. During the ensuing discussion, several participants reiterated the importance of improving the drive lasers. In addition, with the imminent upgrade of the short-pulse laser capability, it will be important to have new diagnostics for characterizing the laser and measuring the laser-produced radiation and energetic particles. Many of these experiments require unique high-precision laser targets. Moreover, MEC can perform 100 shots per day requiring a total of 500 targets per 5-day experimental run. Thus, a reliable source of targets will be very important to the success of MEC laser experiments.

The competitive landscape around LCLS and MEC makes it imperative that experiments are well planned and supported by theoretical modeling and simulations. For this purpose, software packages available from universities, national laboratories and companies need to be made available to users. In addition, future users may find view-factor software that calculates alignment and diagnostic camera outputs specific for each experiment on MEC to be very helpful. 
The last part of the workshop was devoted to ideas for future lasers at LCLS. These include further upgrades of laser power, high repetition rate soft $\mathrm{x}$-ray lasers $(10 \mathrm{~mJ}, 10 \mathrm{~s}$ of $\mathrm{nm}, 100 \mathrm{~Hz}$ ), and laser Spike Trains of Uneven Duration and Delay (STUD). Particularly interesting are high-power lasers approaching and exceeding PW power. The participants preferred PW lasers that would deliver $100 \mathrm{~J}$ or more laser energy on target..

The workshop concluded with the recommendation to hold regular discussion sessions at conferences and meetings to provide the user community with continued input to MEC and LCLS.

Funding for this workshop was provided by DOE Office of Science, Fusion Energy Science, under contract No. AT5015033.

Company sponsors (General Atomics, Amplitude Technologies Coherent, Continuum, GT Advanced Technologies, Imagine Optic, LEO, National Energetics, Northrop Grumman, PRISM Computational Sciences and THALES), and institutes from Lawrence Berkeley National Laboratory, Lawrence Livermore National Laboratory, Stanford and SLAC National Accelerator Laboratory also supported the workshop. More information can be found on the conference website:

http://conf-slac.stanford.edu/hpl-2013/

Roger Falcone Lawrence Berkeley National Laboratory Advanced Light Source and University of California, Berkeley Physics Department Siegfried Glenzer (chair)

SLAC National Accelerator Laboratory Stanford Institute for Materials \& Energy

Sciences

High-Energy-Density Science

Stefan Hau-Riege

Lawrence Livermore National Laboratory Physical and Life Science Directorate $X$-ray Science and Technology 


\section{Figures}

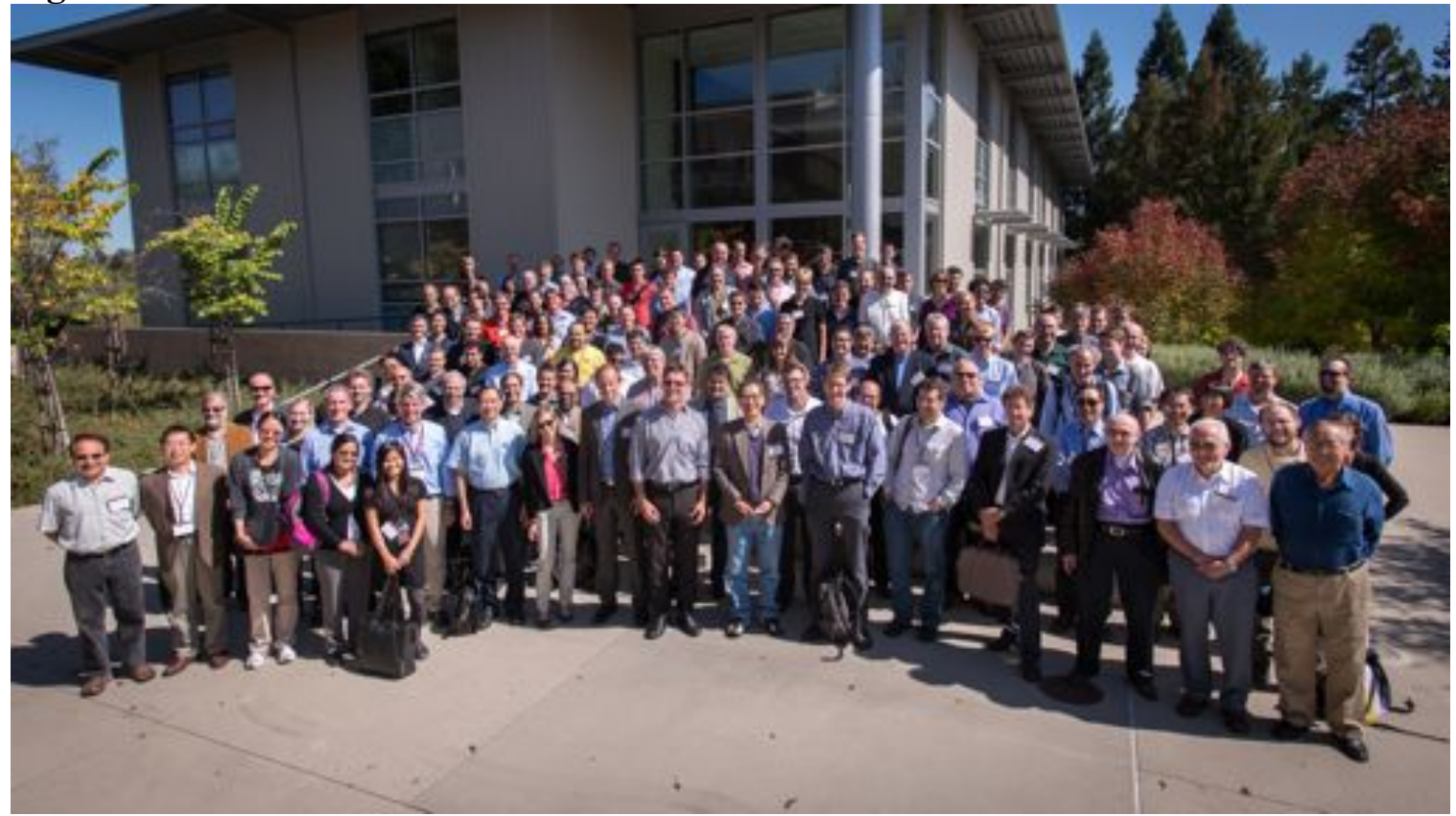

Figure 1: Participants at the User Workshop on High-Power Lasers at the Linac Coherent Light Source, held at SLAC National Accelerator Laboratory, Menlo Park, CA.

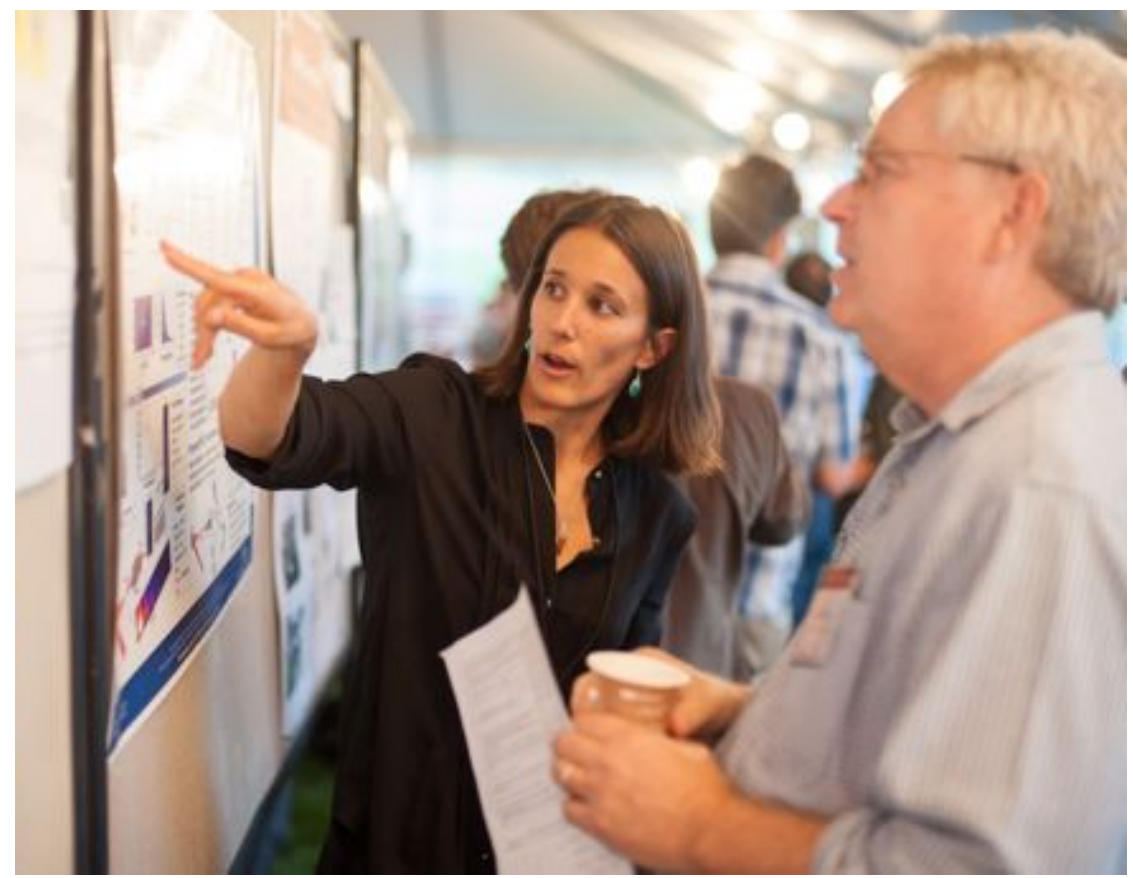

Figure 2: During the poster session, Félicie Albert discusses betatron x-ray production by high-power lasers with Mark Foord (researchers from Lawrence Livermore National Laboratory). 